\title{
Variance-Constrained State Estimation for Networked Multi-rate Systems with Measurement Quantization and Probabilistic Sensor Failures
}

\author{
Yong Zhang*, Zidong Wang and Lifeng Ma
}

\begin{abstract}
This paper is concerned with the variance-constrained state estimation problem for a class of networked multi-rate systems (NMSs) with network-induced probabilistic sensor failures and measurement quantization. The stochastic characteristics of the sensor failures are governed by mutually independent random variables over the interval $[0,1]$. By applying the lifting technique, an augmented system model is established to facilitate the state estimation of the underlying NMSs. With the aid of the stochastic analysis approach, sufficient conditions are derived under which the exponential mean-square stability of the augmented system is guaranteed, the prescribed $H_{\infty}$ performance constraint is achieved and the individual variance constraint on the steady-state estimation error is satisfied. Based on the derived conditions, the addressed variance-constrained state estimation problem of NMSs is recast as a convex optimization one that can be solved via the semi-definite program method. Furthermore, the explicit expression of the desired estimator gains is obtained by means of the feasibility of certain matrix inequalities. Two additional optimization problems are considered with respect to the $H_{\infty}$ performance index and the weighted error variances. Finally, a simulation example is utilized to illustrate the effectiveness of the proposed state estimation method.
\end{abstract}

\section{Index Terms}

Networked multi-rate systems; Variance constraint; $H_{\infty}$ state estimation; Measurement quantization; Probabilistic sensor failures.

\section{INTRODUCTION}

The past few decades have undergone steady revolution leading to new generations of the information and communication technologies. In particular, networked control systems (NCSs) have received considerable research attention because of the urgent need to reduce the cost of installation and facilitate the

This work was supported in part by the National Natural Science Foundation of China under Grants 61104027, 61174107, 61304010 and 61329301, the Royal Society of the U.K., the Natural Science Foundation of Jiangsu Province under Grant BK20130766, the Postdoctoral Science Foundation of China under Grant 2014M551598, the International Postdoctoral Exchange Fellowship from the China Postdoctoral Council, and the Alexander von Humboldt Foundation of Germany.

Y. Zhang is with the School of Information Science and Engineering, Wuhan University of Science and Technology, Wuhan 430081, China. (Email: zhangyong77@wust.edu.cn )

Z. Wang is with the Department of Computer Science, Brunel University London, Uxbridge, Middlesex, UB8 3PH, United Kingdom. He is also with the Communication Systems and Networks (CSN) Research Group, Faculty of Engineering, King Abdulaziz University, Jeddah 21589, Saudi Arabia. (Email: Zidong.Wangebrunel.ac.uk)

L. Ma is with the School of Automation, Nanjing University of Science and Technology, Nanjing 210094, China. (Email: malifeng@njust.edu.cn)

* Corresponding author. E-mail: zhangyong77@wust.edu.cn 
implementation. It is well known that the devices in NCSs are mutually connected via communication networks which are of limited capacity. Therefore, various network-induced phenomena such as communication delay and data missing have inevitably emerged, which should all be taken into account in order to avoid the performance degradation of the NCSs. Consequently, it is not surprising that, the control and filtering/state estimation problems of networked systems with communication delays and/or missing measurements have been reported (see e.g. [4], [7], [10], [11], [13], [22]-[24], [29], [33], [34], [36]).

In computer-based control systems, the interface between the plant and the estimator is often connected via analog-to-digital (A/D) and digital-to-analog (D/A) devices, which normally leads to the quantization process [16]. Actually, quantization error never vanishes when the signals are processed by uniform quantizer [19], [21] or nonuniform quantizer [5], [12], [25], [28], [31]. Accordingly, signal quantization is considered as another source that has significant impact on the achievable performance of the NCSs. It is worth pointing out that, all the references listed above have been concerned with the systems with a single sampling rate. In practice, there are various physical restrictions on the system components such as sensors, actuators, controllers and filters. These physical restrictions include, but are not limited to, the resource constraints on the power, amplitude, frequency, energy and costs, and such restrictions make it extremely difficult to adopt the single-rate sampling strategy for different kinds of devices. Apart from the physical limits, multi-rate sampling also stems from the engineering specifications, for example, sampling the sensor output at a slower rate is often acceptable for cost saving as long as the desired accuracy is ensured. With multi-rate sampling, one could play the trade-offs between the performance index and implementation cost [30]. As such, the multi-rate sampled-data systems have received considerable research interest in the past decades, see [17], [20], [37].

State estimation or filtering has long been a research topic of fundamental importance in signal processing, communications and control applications [1], [3], [8], [9]. Among a variety of existing approaches, the $H_{\infty}$ method has gained particular research attention due to its capability of providing a bound for the worst-case estimation error without the need for knowledge of noise statistics. On the other hand, it is common in practical engineering that the estimation performance requirements are naturally expressed as the upper bounds on estimation error variances [14]. As mentioned in [26], [35], the specified variance constraints may not be minimal, but should meet given engineering requirements. Actually, varianceconstrained control or filtering theory is capable of ensuring the traditional stability with guaranteed upper bounds on the variances of interest and, at the same time, enforcing other performance indices due to its design flexibility. As a result, the variance-constrained theory has been widely applied in solving multi-objective control problems as well as filtering problems, see, e.g. [6], [27], [32].

Summarizing the above discussions, it is of both theoretical importance and practical significance to examine how the inclusion of the multi-rate sampling mechanism would influence the estimation performance of networked multi-rate systems (NMSs). Therefore, the objective of this paper is to design the estimator for a class of NMSs with measurement quantization and probabilistic sensor failures such that the expected exponential mean-square stability, the $H_{\infty}$ performance requirement and the prespecified variance constraints are simultaneously guaranteed. By employing stochastic analysis techniques, the existence of the desired state estimators is investigated and some easy-to-verify sufficient conditions are established. Furthermore, the explicit expression of estimator gain is characterized in terms of the feasibility of certain matrix inequalities. Two additional optimization problems are considered with respect to the 
$H_{\infty}$ performance index and the weighted error variances. Finally, a simulation example is provided to show the effectiveness of the proposed estimator design scheme.

The novelties of this paper lie in the following three aspects: (1) the system model is comprehensive that covers multi-rate sampled-data dynamics, probabilistic sensor failures and measurement quantization, thereby better reflecting the reality within networked environments; (2) by using the lifting technique, the variance-constrained $H_{\infty}$ state estimation problem for NMSs is investigated via a convex optimization technique that caters for sensor failure probabilities and quantization levels; and (3) sufficient conditions are established to quantify the relationships among the $H_{\infty}$ performance, upper bounds on the steady-state estimation error, the quantizer parameters, the statistical information on the sensor failures as well as the multi-rate multiple.

Notation The notation used here is fairly standard except where otherwise stated. $\mathbb{R}^{n}$ and $\mathbb{R}^{n \times m}$ denote, respectively, the $n$-dimensional Euclidean space and the set of all $n \times m$ real matrices. $l_{2}[0, \infty)$ is the space of square summable sequences. The notation $X \geq Y$ (respectively, $X>Y$ ), where $X$ and $Y$ are real symmetric matrices, means that $X-Y$ is positive semi-definite (respectively, positive definite). $\mathbb{E}\{\cdot\}$ stands for the expectation of the stochastic variable ".". 0 and $I$ denote, respectively, the zero matrix of compatible dimensions and the identity matrix of compatible dimensions. In symmetric block matrices or complex matrix expressions, we utilize asterisk $*$ to represent a term that is induced by symmetry, and $\operatorname{diag}\{\cdots\}$ stands for a block-diagonal matrix. $\operatorname{col}\{\cdots\}$ represents a column vector composed of elements. $\|\bullet\|$ refers to the Euclidean norm for vectors.

\section{PROBLEM FORMULATION}

Consider the following class of discrete time systems:

$$
\begin{gathered}
x\left(T_{k+1}\right)=A x\left(T_{k}\right)+B_{1} \omega\left(T_{k}\right)+B_{2} \nu\left(T_{k}\right) \\
z\left(T_{k}\right)=L x\left(T_{k}\right), \quad k=0,1,2, \cdots
\end{gathered}
$$

where $x\left(T_{k}\right) \in \mathbb{R}^{n_{x}}$ represents the state vector, $z\left(T_{k}\right) \in \mathbb{R}^{n_{z}}$ is the signal to be estimated, $\omega\left(T_{k}\right) \in \mathbb{R}^{n_{\omega}}$ is a disturbance input with bounded energy which belongs to $\ell_{2}[0, \infty)$, and $\nu\left(T_{k}\right) \in \mathbb{R}^{n_{\nu}}$ is a zero mean Gaussian white noise sequence with covariance $R>0$.

The measurement with probabilistic sensor failures is described by

$$
y\left(t_{k}\right)=\Xi\left(t_{k}\right) C x\left(t_{k}\right)+D \xi\left(t_{k}\right)=\sum_{s=1}^{m} \beta_{s}\left(t_{k}\right) C_{s} x\left(t_{k}\right)+D \xi\left(t_{k}\right)
$$

where $y\left(t_{k}\right) \in \mathbb{R}^{m}$ is the measured output vector, $\Xi\left(t_{k}\right)$ is a diagonal matrix governing the probabilistic sensor failures described by

$$
\Xi\left(t_{k}\right) \triangleq \operatorname{diag}\left\{\beta_{1}\left(t_{k}\right), \beta_{2}\left(t_{k}\right), \cdots, \beta_{m}\left(t_{k}\right)\right\}
$$

with $\beta_{s}\left(t_{k}\right)(s=1, \ldots, m)$ being $m$ independent random variables which are also independent from $\nu\left(T_{k}\right)$, and the matrix $C_{s}$ is defined by

$$
C_{s} \triangleq \operatorname{diag}\{\underbrace{0, \cdots, 0}_{s-1}, 1, \underbrace{0, \cdots, 0}_{m-s}\} C(s=1,2, \cdots, m) .
$$




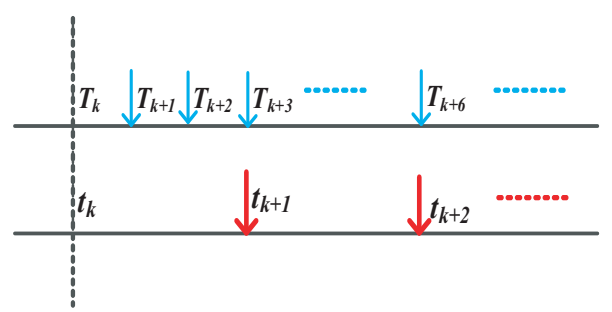

Fig. 1. An example of multi-rate sampled-data systems with $b=3$.

It is assumed that $\beta_{s}\left(t_{k}\right)$ has the probabilistic density function $f\left(\beta_{s}\right)$ on the interval $[0,1]$ with known mathematical expectations $\bar{\beta}_{s}$ and variances $\tilde{\bar{\beta}}_{s}^{2}$. In the sequel, we denote $\Xi \triangleq \mathbb{E}\left[\Xi\left(t_{k}\right)\right]=\operatorname{diag}\left\{\bar{\beta}_{1}, \bar{\beta}_{2}, \cdots, \bar{\beta}_{m}\right\}$. $\xi\left(t_{k}\right) \in \mathbb{R}^{n_{\xi}}$ is the measurement noise which belongings to $\ell_{2}[0, \infty) . A, B_{1}, B_{2}, L, C$ and $D$ are known matrices with appropriate dimensions.

Remark 1: Note that an increasing number of sensors have been installed to structures for monitoring and control, and sensor faults become more frequent compared to the structure's lifetime [18]. For small autonomous helicopters, the fault detection problem has been investigated in [15], where several sensor failures have been considered such as total sensor failure, stuck with constant bias sensor failure, drift or additive-type sensor failure, multiplicative-type sensor failure and outlier data sensor failure. In Eq. (3), the random variable $\beta_{s}\left(t_{k}\right)$ taking value on the interval $\left[\begin{array}{ll}0 & 1\end{array}\right]$ is introduced to describe possible failure for the $s$-th $(s=1, \ldots, m)$ sensor, and this kind of measurement model can include the Bernoulli distribution model [36] as a special case. Actually, this sensor fault model accounts for the multiplicative-type sensor failures that have been discussed in [15].

For a given frame period $h$, we make the following assumptions about the sampling period for (1)-(3). Assumption 1: The system state $x\left(T_{k}\right)$ and its estimation $z\left(T_{k}\right)$ are updated at instants $T_{k}$, and $T_{k+1}-$ $T_{k} \triangleq h, k=0,1,2, \cdots$.

Assumption 2: The measurement $y\left(t_{k}\right)$ from the system is sampled at instants $t_{k}$ and $t_{k+1}-t_{k} \triangleq$ $b h, k=0,1,2, \cdots$, where $b$ is a positive integer.

It can be seen that (1) and (2) evolve with a faster sampling period $h$, while the measurement dynamics (3) is generated with a slower period $b h$, i.e. the measurement sampling periods $t_{k+1}-t_{k}$ are integer multiples of the period $h$. Accordingly, (1)-(3) is essentially a multi-rate sampled-data (MRSD) system model. An illustration of MRSD is shown in Fig. 1 with $b=3$, where the system state and estimation signal are updating with period $h$, and the measurements are sampled with period $3 h$.

In this paper, the quantization effect on measurement $y\left(t_{k}\right)$ is considered with the map of the quantization process given by

$$
\bar{y}\left(t_{k}\right)=q\left(y\left(t_{k}\right)\right)=\operatorname{col}\left\{q_{1}\left(y^{(1)}\left(t_{k}\right)\right), q_{2}\left(y^{(2)}\left(t_{k}\right)\right), \cdots, q_{m}\left(y^{(m)}\left(t_{k}\right)\right)\right\}
$$

where $\bar{y}\left(t_{k}\right)$ is the signal after quantization. The quantizer $q(\cdot)$ is assumed to be of the logarithmic type, that is, for each $q_{s}\left(y^{(s)}\left(t_{k}\right)\right)(s=1,2, \cdots, m)$, the set of quantization levels is described by

$$
\Im=\left\{ \pm u_{i}^{(s)}, u_{i}^{(s)}=\left(\chi^{(s)}\right)^{i} u_{0}^{(s)}, i=0, \pm 1, \pm 2, \cdots\right\} \bigcup\{0\}, \quad 0<\chi^{(s)}<1, u_{0}^{(s)}>0
$$

where $\chi^{(s)}(s=1,2, \cdots, m)$ is called the quantization density. Each of the quantization level corresponds to a segment such that the quantizer maps the whole segment to this quantization level. The logarithmic 
quantizer $q_{s}(\cdot)$ is defined as

$$
q_{s}\left(y^{(s)}\left(t_{k}\right)\right)= \begin{cases}u_{i}^{(s)}, & \frac{1}{1+\delta_{s}} u_{i}^{(s)}<y^{(s)}\left(t_{k}\right)<\frac{1}{1-\delta_{s}} u_{i}^{(s)} \\ 0, & y^{(s)}\left(t_{k}\right)=0 \\ -q_{s}\left(-y^{(s)}\left(t_{k}\right)\right), & y^{(s)}\left(t_{k}\right)<0\end{cases}
$$

where $\delta_{s}=\frac{1-\chi^{(s)}}{1+\chi^{(s)}}$. It can be easily observed from the above definition that $q_{s}\left(y^{(s)}\left(t_{k}\right)\right)=\left(1+\triangle^{(s)}\left(t_{k}\right)\right) y^{(s)}\left(t_{k}\right)$ holds for certain $\triangle^{(s)}\left(t_{k}\right)$ satisfying $\triangle^{(s)}\left(t_{k}\right) \leq \delta_{s}$.

According to the above transformation, the quantization effects have been transformed into sectorbounded uncertainties [12]. Defining $\triangle\left(t_{k}\right)=\operatorname{diag}\left\{\triangle^{(1)}\left(t_{k}\right), \triangle^{(2)}\left(t_{k}\right), \cdots, \triangle^{(m)}\left(t_{k}\right)\right\}$, the measurement after quantization can be expressed as

$$
\bar{y}\left(t_{k}\right)=\left(I+\triangle\left(t_{k}\right)\right) y\left(t_{k}\right)
$$

Denoting $\Lambda \triangleq \operatorname{diag}\left\{\delta_{1}, \delta_{2}, \cdots, \delta_{m}\right\}$ and setting $F\left(t_{k}\right) \triangleq \triangle\left(t_{k}\right) \Lambda^{-1}$, we can know that $F\left(t_{k}\right)$ is a realvalued time-varying matrix satisfying $F\left(t_{k}\right)^{T} F\left(t_{k}\right)=F\left(t_{k}\right) F\left(t_{k}\right)^{T} \leq I$.

Note that it is mathematically difficult to handle the variance-constrained state estimation problem directly for such kind of MRSD system. In the next section, we are going to convert the resulting MRSD system into a single-rate system for technical convenience.

By applying the relation (1) recursively, one obtains the following equations with time scale $t_{k}$ :

$$
\left\{\begin{aligned}
x\left(t_{k+1}\right) & =A^{b} x\left(t_{k}\right)+\bar{B}_{1,1} \bar{\omega}\left(t_{k}\right)+\bar{B}_{2,1} \bar{\nu}\left(t_{k}\right) \\
x\left(t_{k+1}-h\right) & =x\left(t_{k}+(b-1) h\right)=A^{b-1} x\left(t_{k}\right)+\bar{B}_{1,2} \bar{\omega}\left(t_{k}\right)+\bar{B}_{2,2} \bar{\nu}\left(t_{k}\right) \\
& \vdots \\
x\left(t_{k+1}-(b-1) h\right) & =x\left(t_{k}+h\right)=A x\left(t_{k}\right)+\bar{B}_{1, b} \bar{\omega}\left(t_{k}\right)+\bar{B}_{2, b} \bar{\nu}\left(t_{k}\right)
\end{aligned}\right.
$$

where

$$
\begin{aligned}
& \bar{\omega}\left(t_{k}\right) \triangleq \operatorname{col}\left\{\omega\left(t_{k}\right), \omega\left(t_{k}+h\right), \cdots, \omega\left(t_{k}+(b-1) h\right)\right\}, \\
& \bar{\nu}\left(t_{k}\right) \triangleq \operatorname{col}\left\{\nu\left(t_{k}\right), \nu\left(t_{k}+h\right), \cdots, \nu\left(t_{k}+(b-1) h\right)\right\}, \\
& \bar{B}_{j, 1} \triangleq\left[\begin{array}{lllll}
A^{b-1} B_{j} & A^{b-2} B_{j} & \cdots & A B_{j} & B_{j}
\end{array}\right], \\
& \bar{B}_{j, 2} \triangleq\left[\begin{array}{lllll}
A^{b-2} B_{j} & A^{b-3} B_{j} & \cdots & B_{j} & 0
\end{array}\right], \cdots, \\
& \bar{B}_{j, b-1} \triangleq\left[\begin{array}{lll}
A B_{j} & B_{j} \underbrace{0 \cdots 0}_{b-2}
\end{array}\right], \quad \bar{B}_{j, b} \triangleq[B_{j} \underbrace{0 \cdots 0}_{b-1}],(j=1,2) .
\end{aligned}
$$

Based on the quantized measurement signal $\bar{y}\left(t_{k}\right)$ and system (5), the following estimator is constructed:

$$
\left\{\begin{aligned}
\hat{x}\left(t_{k+1}\right) & =A^{b} \hat{x}\left(t_{k}\right)+H_{1}\left(\bar{y}\left(t_{k}\right)-\bar{\Xi} C \hat{x}\left(t_{k}\right)\right) \\
\hat{x}\left(t_{k+1}-h\right) & =A^{b-1} \hat{x}\left(t_{k}\right)+H_{2}\left(\bar{y}\left(t_{k}\right)-\bar{\Xi} C \hat{x}\left(t_{k}\right)\right) \\
& \vdots \\
\hat{x}\left(t_{k+1}-(b-1) h\right) & =A \hat{x}\left(t_{k}\right)+H_{b}\left(\bar{y}\left(t_{k}\right)-\bar{\Xi} C \hat{x}\left(t_{k}\right)\right) \\
\hat{z}\left(t_{k}-i h\right) & =L \hat{x}\left(t_{k}-i h\right),(i=0,1,2, \cdots, b-1)
\end{aligned}\right.
$$

where $\hat{x}\left(t_{k}-i h\right) \in \mathbb{R}^{n_{x}}(i=0,1,2, \cdots, b-1)$ are the estimated state, $\hat{z}\left(t_{k}-i h\right) \in \mathbb{R}^{n_{z}}(i=0,1,2, \cdots, b-$ 1) are the estimated output, and $H_{\varrho}(\varrho=1,2, \cdots, b)$ are the estimator gains to be designed. 


\section{Denoting}

$$
\begin{aligned}
& \eta\left(t_{k}\right) \triangleq \operatorname{col}\left\{x\left(t_{k}\right), x\left(t_{k}-h\right), \cdots, x\left(t_{k}-(b-1) h\right)\right\}, e\left(t_{k}-i h\right) \triangleq x\left(t_{k}-i h\right)-\hat{x}\left(t_{k}-i h\right), \\
& e_{\eta}\left(t_{k}\right) \triangleq \operatorname{col}\left\{e\left(t_{k}\right), e\left(t_{k}-h\right), \cdots, e\left(t_{k}-(b-1) h\right)\right\}, e_{z}\left(t_{k}-i h\right) \triangleq z\left(t_{k}-i h\right)-\hat{z}\left(t_{k}-i h\right), \\
& \tilde{z}_{e}\left(t_{k}\right) \triangleq \operatorname{col}\left\{e_{z}\left(t_{k}\right), e_{z}\left(t_{k}-h\right), \cdots, e_{z}\left(t_{k}-(b-1) h\right)\right\}, \tilde{\beta}_{s}\left(t_{k}\right) \triangleq \beta_{s}\left(t_{k}\right)-\bar{\beta}_{s}, \\
& d\left(t_{k}\right) \triangleq \operatorname{col}\left\{\xi\left(t_{k}\right), \bar{\omega}\left(t_{k}\right)\right\}, \bar{A}_{1} \triangleq \operatorname{col}\left\{A^{b}, A^{b-1}, \cdots, A\right\}, \quad \hat{A} \triangleq[\bar{A}_{1} \underbrace{0 \cdots 0}_{b-1}], \\
& \hat{H} \triangleq \operatorname{col}\left\{H_{1}, H_{2}, \cdots, H_{b}\right\}, \mathcal{I} \triangleq[I \underbrace{0 \cdots 0}_{b-1}], \mathcal{A} \triangleq \hat{A}-\hat{H} \bar{\Xi} C \mathcal{I}, \mathcal{C} \triangleq-\hat{H} F\left(t_{k}\right) \Lambda \Xi C \mathcal{I}, \\
& \overline{\mathcal{A}}_{s} \triangleq-\hat{H} C_{s} \mathcal{I}, \overline{\mathcal{C}}_{s} \triangleq-\hat{H} F\left(t_{k}\right) \Lambda C_{s} \mathcal{I}, \hat{B}_{j} \triangleq \operatorname{col}\left\{\bar{B}_{j, 1}, \bar{B}_{j, 2}, \cdots, \bar{B}_{j, b-1}, \bar{B}_{j, b}\right\}, \\
& \mathcal{B}_{1} \triangleq\left[\begin{array}{ll}
-\hat{H} D & \hat{B}_{1}
\end{array}\right], \overline{\mathcal{B}}_{1} \triangleq\left[\begin{array}{ll}
-\hat{H} F\left(t_{k}\right) \Lambda D & 0
\end{array}\right], \mathcal{B}_{2} \triangleq \hat{B}_{2}, \hat{L} \triangleq \operatorname{diag}\{\underbrace{L, L, \cdots, L}_{b}\}, \\
& (i=0,1,2, \cdots, b-1 ; j=1,2 ; s=1,2, \cdots, m),
\end{aligned}
$$

and using the lifting technique, the dynamics of estimation error can be obtained from (5) and (6) as follows:

$$
\left\{\begin{aligned}
e_{\eta}\left(t_{k+1}\right) & =\mathcal{A} e_{\eta}\left(t_{k}\right)+\left\{\mathcal{C}+\sum_{s=1}^{m} \tilde{\beta}_{s}\left(t_{k}\right)\left(\overline{\mathcal{A}}_{s}+\overline{\mathcal{C}}_{s}\right)\right\} \eta\left(t_{k}\right)+\left(\mathcal{B}_{1}+\overline{\mathcal{B}}_{1}\right) d\left(t_{k}\right)+\mathcal{B}_{2} \bar{\nu}\left(t_{k}\right) \\
\tilde{z}_{e}\left(t_{k}\right) & =\hat{L} e_{\eta}\left(t_{k}\right)
\end{aligned}\right.
$$

and $\bar{\nu}\left(t_{k}\right)$ satisfies the following relationship:

$$
\mathbb{E}\left\{\bar{\nu}\left(t_{k}\right)\right\}=0, \quad \mathbb{E}\left\{\bar{\nu}\left(t_{k}\right) \bar{\nu}^{T}\left(t_{i}\right)\right\}=0(k \neq i), \mathbb{E}\left\{\bar{\nu}\left(t_{k}\right) \bar{\nu}^{T}\left(t_{k}\right)\right\}=\operatorname{diag}\{\underbrace{R, \cdots, R}_{b}\} \triangleq \mathcal{R} .
$$

By denoting $\tilde{e}\left(t_{k}\right) \triangleq \operatorname{col}\left\{e_{\eta}, \eta\left(t_{k}\right)\right\}, \bar{H} \triangleq \operatorname{col}\{-\hat{H}, 0\}, \bar{C} \triangleq\left[\begin{array}{cc}0 & \Lambda \Xi C \mathcal{I}\end{array}\right], \bar{C}_{s} \triangleq\left[\begin{array}{ll}0 & C_{s} \mathcal{I}\end{array}\right], \bar{D} \triangleq$

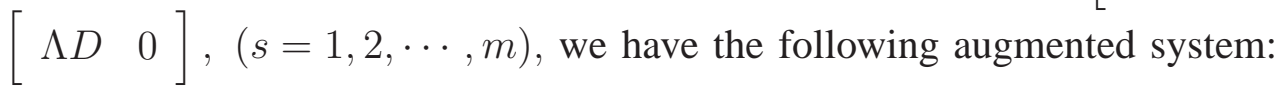

$$
\left\{\begin{aligned}
\tilde{e}\left(t_{k+1}\right) & =\left\{(\mathscr{A}+\mathscr{C})+\sum_{s=1}^{m} \tilde{\beta}_{s}\left(t_{k}\right)\left(\overline{\mathscr{A}}_{s}+\overline{\mathscr{C}}_{s}\right)\right\} \tilde{e}\left(t_{k}\right)+\left(\mathscr{B}_{1}+\overline{\mathscr{B}}_{1}\right) d\left(t_{k}\right)+\mathscr{B}_{2} \bar{\nu}\left(t_{k}\right) \\
\tilde{z}_{e}\left(t_{k}\right) & =\mathscr{L} \tilde{e}\left(t_{k}\right)
\end{aligned}\right.
$$

where

$$
\begin{aligned}
& \mathscr{A} \triangleq \operatorname{diag}\{\mathcal{A}, \hat{A}\}, \mathscr{C} \triangleq \bar{H} F\left(t_{k}\right) \bar{C}, \overline{\mathscr{A}}_{s} \triangleq \bar{H} \bar{C}_{s}, \overline{\mathscr{C}}_{s} \triangleq \bar{H} F\left(t_{k}\right) \Lambda \bar{C}_{s}(s=1,2, \cdots, m), \\
& \mathscr{B}_{1} \triangleq\left[\begin{array}{cc}
-\hat{H} D & \hat{B}_{1} \\
0 & \hat{B}_{1}
\end{array}\right], \mathscr{B}_{2} \triangleq\left[\begin{array}{l}
\hat{B}_{2} \\
\hat{B}_{2}
\end{array}\right], \overline{\mathscr{B}}_{1} \triangleq \bar{H} F\left(t_{k}\right) \bar{D}, \mathscr{L} \triangleq\left[\begin{array}{lll}
\hat{L} & \underbrace{0 \cdots}_{b}
\end{array}\right] .
\end{aligned}
$$

Remark 2: So far, by using the lifting technique, the model (9) for NMSs have been obtained. Comparing with the models of NMSs in [20], [37], model (9) exhibits two distinguished features: i) both the quantization and probabilistic sensor failures are considered and therefore the model (9) is quite comprehensive to better reflect the networked environment; ii) the coefficients in model (3) are governed by individual random variables taking value on $[0,1]$ and such representations include the widely studied Bernoulli distribution (see e.g. [20], [37]) as a special case.

Before proceeding further, we introduce the following definition. 
Definition 1: [36] The augmented system (9) is said to be exponentially mean-square stable if, with $d\left(t_{k}\right)=0$ and $\bar{\nu}\left(t_{k}\right)=0$, there exist constants $\alpha \geq 1$ and $\hbar \in(0,1)$ such that

$$
\mathbb{E}\left\{\left\|\tilde{e}\left(t_{k}\right)\right\|^{2}\right\} \leq \alpha \hbar^{t_{k}} \mathbb{E}\left\{\left\|\tilde{e}\left(t_{0}\right)\right\|^{2}\right\}
$$

The main purpose of this paper is to design the estimator in the form of (6) such that the following requirements are satisfied simultaneously:

(a) the augmented system (9) is exponentially mean-square stable;

(b) under zero-initial condition, the estimation error $\tilde{z}_{e}\left(t_{k}\right)$ with respect to the energy bounded disturbance $d\left(t_{k}\right)$ satisfies

$$
\sum_{k=0}^{\infty} \mathbb{E}\left\{\left\|\tilde{z}_{e}\left(t_{k}\right)\right\|^{2}\right\}<\gamma^{2} \sum_{k=0}^{\infty} \mathbb{E}\left\{\left\|d\left(t_{k}\right)\right\|^{2}\right\}
$$

where $\gamma$ is a given disturbance attenuation level;

(c) the individual steady-state estimation error variance $\mathcal{E}_{e}^{(r)}$ satisfies

$$
\mathcal{E}_{e}^{(r)} \triangleq \lim _{k \rightarrow \infty} \mathbb{E}\left\{e_{r}\left(t_{k}\right) e_{r}^{T}\left(t_{k}\right)\right\} \leq \sigma_{r}^{2}\left(r=1,2, \cdots, n_{x}\right)
$$

where $e_{r}\left(t_{k}\right)$ is the $r$ th entry of the vector $e\left(t_{k}\right), \mathcal{E}_{e}^{(r)}$ stands for the steady-state variance of the $r$ th state estimation error and $\sigma_{r}^{2}>0$ denotes the prespecified variance constraint on steady-state estimation error $e_{r}\left(t_{k}\right)\left(r=1,2, \cdots, n_{x}\right)$.

\section{MAIN RESUlTS}

\section{A. $H_{\infty}$ Performance}

The following theorem gives a sufficient condition for the exponential mean-square stability as well as $H_{\infty}$ performance constraint of the augmented system (9).

Theorem 1: For the given disturbance attenuation level $\gamma>0$ and estimator gain $H$, the augmented system (9) is exponentially mean-square stable and simultaneously satisfies the $H_{\infty}$ performance constraint (10) if there exists a positive definite matrix $\mathcal{P}$ such that the following matrix inequality

$$
\Phi \triangleq\left[\begin{array}{cc}
\Gamma+\mathscr{L}^{T} \mathscr{L} & (\mathscr{A}+\mathscr{C})^{T} \mathcal{P}\left(\mathscr{B}_{1}+\overline{\mathscr{B}}_{1}\right) \\
* & \left(\mathscr{B}_{1}+\overline{\mathscr{B}}_{1}\right)^{T} \mathcal{P}\left(\mathscr{B}_{1}+\overline{\mathscr{B}}_{1}\right)-\gamma^{2} I
\end{array}\right]<0
$$

holds, where $\Gamma \triangleq(\mathscr{A}+\mathscr{C})^{T} \mathcal{P}(\mathscr{A}+\mathscr{C})+\sum_{s=1}^{m} \tilde{\bar{\beta}}_{s}^{2}\left(\overline{\mathscr{A}}_{s}+\overline{\mathscr{C}}_{s}\right)^{T} \mathcal{P}\left(\overline{\mathscr{A}}_{s}+\overline{\mathscr{C}}_{s}\right)-\mathcal{P}$.

Proof: Choose the following Lyapunov function:

$$
V\left(\tilde{e}\left(t_{k}\right)\right)=\tilde{e}^{T}\left(t_{k}\right) \mathcal{P} \tilde{e}\left(t_{k}\right) .
$$

By calculating the difference of $V\left(\tilde{e}\left(t_{k}\right)\right)$ along the trajectory of the augmented system (9) with $d\left(t_{k}\right)=0$ and $\bar{\nu}\left(t_{k}\right)=0$, and taking the mathematical expectation, one has

$$
\begin{aligned}
\mathbb{E}\left\{\triangle V\left(\tilde{e}\left(t_{k}\right)\right)\right\} & =\mathbb{E}\left\{\tilde{e}^{T}\left(t_{k+1}\right) \mathcal{P} \tilde{e}\left(t_{k+1}\right)-\tilde{e}^{T}\left(t_{k}\right) \mathcal{P} \tilde{e}\left(t_{k}\right)\right\} \\
& =\mathbb{E}\left\{\tilde { e } ^ { T } ( t _ { k } ) \left[\left((\mathscr{A}+\mathscr{C})+\sum_{s=1}^{m} \tilde{\beta}_{s}\left(t_{k}\right)\left(\overline{\mathscr{A}}_{s}+\overline{\mathscr{C}}_{s}\right)^{T} \mathcal{P}\right.\right.\right.
\end{aligned}
$$




$$
\begin{aligned}
& \left.\left.\times\left((\mathscr{A}+\mathscr{C})+\sum_{s=1}^{m} \tilde{\beta}_{s}\left(t_{k}\right)\left(\overline{\mathscr{A}}_{s}+\overline{\mathscr{C}}_{s}\right)\right)-\mathcal{P}\right] \tilde{e}\left(t_{k}\right)\right\} \\
= & \tilde{e}^{T}\left(t_{k}\right)\left\{(\mathscr{A}+\mathscr{C})^{T} \mathcal{P}(\mathscr{A}+\mathscr{C})+\sum_{s=1}^{m} \tilde{\bar{\beta}}_{s}^{2}\left(\overline{\mathscr{A}}_{s}+\overline{\mathscr{C}}_{s}\right)^{T} \mathcal{P}\left(\overline{\mathscr{A}}_{s}+\overline{\mathscr{C}}_{s}\right)-\mathcal{P}\right\} \tilde{e}\left(t_{k}\right) \\
= & \tilde{e}^{T}\left(t_{k}\right) \Gamma \tilde{e}\left(t_{k}\right) .
\end{aligned}
$$

We can obtain from (12) that $\Gamma<0$ and, subsequently,

$$
\mathbb{E}\left\{\triangle V\left(\tilde{e}\left(t_{k}\right)\right)\right\} \leq-\lambda_{\min }(-\Gamma)\left\|\tilde{e}\left(t_{k}\right)\right\|^{2} .
$$

Hence, by following the similar analysis in [38], the augmented system (9) is exponentially mean-square stable.

Next, based on the zero initial condition, let us establish the $H_{\infty}$ performance constraint of augmented system (9) with $\bar{\nu}\left(t_{k}\right)=0$ by the following derivation:

$$
\begin{aligned}
\mathbb{E}\left\{\triangle V\left(\tilde{e}\left(t_{k}\right)\right)\right\}+ & \mathbb{E}\left\{\tilde{z}_{e}^{T}\left(t_{k}\right) \tilde{z}_{e}\left(t_{k}\right)\right\}-\gamma^{2} \mathbb{E}\left\{d^{T}\left(t_{k}\right) d\left(t_{k}\right)\right\} \\
= & \tilde{e}^{T}\left(t_{k}\right)\left\{\Gamma+\mathscr{L}^{T} \mathscr{L}\right\} \tilde{e}\left(t_{k}\right)+2 \tilde{e}^{T}\left(t_{k}\right)\left\{(\mathscr{A}+\mathscr{C})^{T} \mathcal{P}\left(\mathscr{B}_{1}+\overline{\mathscr{B}}_{1}\right)\right\} d\left(t_{k}\right) \\
& +d^{T}\left(t_{k}\right)\left\{\left(\mathscr{B}_{1}+\overline{\mathscr{B}}_{1}\right)^{T} \mathcal{P}\left(\mathscr{B}_{1}+\overline{\mathscr{B}}_{1}\right)-\gamma^{2} I\right\} d\left(t_{k}\right) \\
= & \vartheta^{T}\left(t_{k}\right) \Phi \vartheta\left(t_{k}\right)
\end{aligned}
$$

where $\vartheta\left(t_{k}\right) \triangleq \operatorname{col}\left\{\tilde{e}\left(t_{k}\right), d\left(t_{k}\right)\right\}$. Furthermore, by using the Schur Complement Lemma to (12), we have $\Phi<0$ implying

$$
\mathbb{E}\left\{\triangle V\left(\tilde{e}\left(t_{k}\right)\right)\right\}+\mathbb{E}\left\{\tilde{z}_{e}^{T}\left(t_{k}\right) \tilde{z}_{e}\left(t_{k}\right)\right\}-\gamma^{2} \mathbb{E}\left\{d^{T}\left(t_{k}\right) d\left(t_{k}\right)\right\}<0
$$

for all nonzero $d\left(t_{k}\right)$.

By considering the zero initial condition, the above inequality indicates that

$$
\sum_{k=0}^{\infty} \mathbb{E}\left\{\tilde{z}_{e}^{T}\left(t_{k}\right) \tilde{z}_{e}\left(t_{k}\right)\right\}<\gamma^{2} \sum_{k=0}^{\infty} \mathbb{E}\left\{d^{T}\left(t_{k}\right) d\left(t_{k}\right)\right\}
$$

which is equivalent to (10). The proof of this theorem is now complete.

\section{B. Variance Analysis}

The following theorem presents sufficient conditions that guarantee the exponential mean-square stability of the augmented system (9) and, at the same time, enforce the individual steady-state estimation error variance constraints.

Theorem 2: For the given steady-state variance upper bounds $\sigma_{r}^{2}\left(r=1,2, \cdots, n_{x}\right)$ and estimator gain $H$, the augmented system (9) is exponentially mean-square stable and simultaneously satisfies the steadystate variance constraint (11) if there exists a positive definite matrix $\mathcal{Q}$ such that the following matrix inequalities

$$
\begin{gathered}
(\mathscr{A}+\mathscr{C}) \mathcal{Q}(\mathscr{A}+\mathscr{C})^{T}+\sum_{s=1}^{m} \tilde{\bar{\beta}}_{s}^{2}\left(\overline{\mathscr{A}}_{s}+\overline{\mathscr{C}}_{s}\right) \mathcal{Q}\left(\overline{\mathscr{A}}_{s}+\overline{\mathscr{C}}_{s}\right)^{T}-\mathcal{Q}+\mathscr{B}_{2} \mathcal{R} \mathscr{B}_{2}^{T}<0 \\
\underline{\mathcal{I}}_{r} \overline{\mathcal{I}}_{\ell} \mathcal{Q} \overline{\mathcal{I}}_{\ell}^{T} \underline{\mathcal{I}}_{r}^{T} \leq \sigma_{r}^{2} \quad\left(r=1,2, \cdots, n_{x} ; \ell=1,2, \cdots, b\right)
\end{gathered}
$$


hold, where $\overline{\mathcal{I}}_{\ell}=\left[\begin{array}{llll}\underbrace{0 \cdots 0}_{\ell-1} & I \underbrace{0 \cdots 0}_{2 b-\ell}\end{array}\right]$ and $\underline{\mathcal{I}}_{r}=\left[\begin{array}{llll}\underbrace{0 \cdots 0}_{r-1} & 1 & \underbrace{0 \cdots 0}_{n_{x}-r}\end{array}\right]$.

Proof: First of all, it follows from (16) that

$$
(\mathscr{A}+\mathscr{C}) \mathcal{Q}(\mathscr{A}+\mathscr{C})^{T}+\sum_{s=1}^{m} \tilde{\bar{\beta}}_{s}^{2}\left(\overline{\mathscr{A}}_{s}+\overline{\mathscr{C}}_{s}\right) \mathcal{Q}\left(\overline{\mathscr{A}}_{s}+\overline{\mathscr{C}}_{s}\right)^{T}-\mathcal{Q}<-\mathscr{B}_{2} \mathcal{R} \mathscr{B}_{2}^{T}<0 .
$$

Based on (18, it can be inferred from [35] that the augmented system (9) is exponentially mean-square stable and, subsequently, the steady-state covariance $\hat{\mathcal{Q}}$ defined by

$$
\hat{\mathcal{Q}} \triangleq \lim _{k \rightarrow \infty} \mathbb{E}\left\{\tilde{e}\left(t_{k}\right) \tilde{e}^{T}\left(t_{k}\right)\right\} .
$$

exists and satisfies the following discrete-time modified Lyapunov equation:

$$
(\mathscr{A}+\mathscr{C}) \hat{\mathcal{Q}}(\mathscr{A}+\mathscr{C})^{T}+\sum_{s=1}^{m} \tilde{\bar{\beta}}_{s}^{2}\left(\overline{\mathscr{A}}_{s}+\overline{\mathscr{C}}_{s}\right) \hat{\mathcal{Q}}\left(\overline{\mathscr{A}}_{s}+\overline{\mathscr{C}}_{s}\right)^{T}-\hat{\mathcal{Q}}+\mathscr{B}_{2} \mathcal{R} \mathscr{B}_{2}^{T}=0
$$

Subtracting (20) from (18) gives

$$
(\mathscr{A}+\mathscr{C})(\mathcal{Q}-\hat{\mathcal{Q}})(\mathscr{A}+\mathscr{C})^{T}+\sum_{s=1}^{m} \tilde{\bar{\beta}}_{s}^{2}\left(\overline{\mathscr{A}}_{s}+\overline{\mathscr{C}}_{s}\right)(\mathcal{Q}-\hat{\mathcal{Q}})\left(\overline{\mathscr{A}}_{s}+\overline{\mathscr{C}}_{s}\right)^{T}-(\mathcal{Q}-\hat{\mathcal{Q}})<0
$$

which indicates from [35] that $\mathcal{Q}-\hat{\mathcal{Q}} \geq 0$.

Finally, considering the definitions of (11) and (19), we can obtain that

$$
\begin{aligned}
\mathcal{E}_{e}^{(r)} & \triangleq \lim _{k \rightarrow \infty} \mathbb{E}\left\{e_{r}\left(t_{k}\right) e_{r}^{T}\left(t_{k}\right)\right\} \triangleq \lim _{k \rightarrow \infty} \mathbb{E}\left\{\underline{\mathcal{I}}_{r} e\left(t_{k}\right) e^{T}\left(t_{k}\right) \underline{\mathcal{I}}_{r}^{T}\right\} \\
& \triangleq \underline{\mathcal{I}}_{r}\left\{\lim _{k \rightarrow \infty} \mathbb{E}\left\{\overline{\mathcal{I}}_{\ell} \tilde{e}\left(t_{k}\right) \tilde{e}^{T}\left(t_{k}\right) \overline{\mathcal{I}}_{\ell}^{T}\right\}\right\} \underline{\mathcal{I}}_{r}^{T} \\
& \triangleq \underline{\mathcal{I}}_{r} \overline{\mathcal{I}}_{\ell} \hat{\mathcal{Q}} \overline{\mathcal{I}}_{\ell}^{T} \underline{\mathcal{I}}_{r}^{T} \leq \underline{\mathcal{I}}_{r} \overline{\mathcal{I}}_{\ell} \mathcal{Q} \overline{\mathcal{I}}_{\ell}^{T} \underline{\mathcal{I}}_{r}^{T}
\end{aligned}
$$

Therefore, matrix inequality (17) indicates that the requirement (c) is also met and the proof is now complete.

To conclude the above analysis, we present a theorem which intends to take both the $H_{\infty}$ performance and the variance constraint into consideration in a unified framework. Before giving our main result, we introduce the following well-known lemma.

Lemma 1: [2] Let $\Omega=\Omega^{T}, S$ and $U$ be real matrices with appropriate dimensions, and matrix $F(\cdot)$ satisfies $F(\cdot) F^{T}(\cdot) \leq I$, then

$$
\Omega+U F(\cdot) M+M^{T} F^{T}(\cdot) U^{T}<0
$$

if and only if there exists a positive scalar $\varepsilon$ such that

$$
\Omega+\frac{1}{\varepsilon} U U^{T}+\varepsilon M^{T} M<0
$$

or equivalently

$$
\left[\begin{array}{ccc}
\Omega & U & \varepsilon M^{T} \\
* & -\varepsilon I & 0 \\
* & * & -\varepsilon I
\end{array}\right]<0
$$

For convenience of later development, we denote

$$
\breve{\mathcal{P}} \triangleq \operatorname{diag}\left\{P_{1}, P_{2}, \cdots, P_{2 b}\right\}, \breve{\mathscr{P}} \triangleq \operatorname{diag}\{\underbrace{\breve{\mathcal{P}}, \cdots, \breve{\mathcal{P}}}_{m}\}, \mathcal{W} \triangleq \operatorname{diag}\{\underbrace{W, W, \cdots, W}_{b}\}
$$




$$
\begin{aligned}
& \mathscr{W} \triangleq \operatorname{diag}\{\mathcal{W}, \mathcal{W}\}, \hat{\mathcal{H}} \triangleq \operatorname{col}\left\{\mathcal{H}_{1}, \cdots, \mathcal{H}_{b}\right\}, \underline{\mathcal{H}}_{s} \triangleq \operatorname{col}\{\underbrace{0, \cdots, 0}_{s-1},-\hat{\mathcal{H}}, \underbrace{0, \cdots, 0}_{m-s}\} \\
& \underline{\mathcal{H}} \triangleq \operatorname{col}\{0,0,0,-\hat{\mathcal{H}}\}, \mathscr{W} \mathscr{A}=\operatorname{diag}\{\mathcal{W} \hat{A}-\hat{\mathcal{H}} \bar{\Xi} C \mathcal{I}, \mathcal{W} \hat{A}\}, \mathscr{W} \overline{\mathscr{A}}_{s}=\left[\begin{array}{cc}
0 & -\hat{\mathcal{H}} C_{s} \mathcal{I} \\
0 & 0
\end{array}\right], \\
& \mathscr{W} \mathscr{B}_{1}=\left[\begin{array}{cc}
-\hat{\mathcal{H}} D & \mathcal{W} \hat{B}_{1} \\
0 & \mathcal{W} \hat{B}_{1}
\end{array}\right], \quad \hat{\mathcal{X}}^{T} \triangleq\left[\begin{array}{llll}
\tilde{\beta}_{1}\left(\mathscr{W} \overline{\mathscr{A}}_{1}\right)^{T} & \tilde{\bar{\beta}}_{2}\left(\mathscr{W} \overline{\mathscr{A}}_{2}\right)^{T} & \ldots & \tilde{\bar{\beta}}_{m}\left(\mathscr{W} \overline{\mathscr{A}}_{m}\right)^{T}
\end{array}\right], \\
& \hat{\bar{\Sigma}}_{1} \triangleq\left[\begin{array}{cc}
\hat{\bar{\Sigma}}_{1} & \hat{\bar{\Sigma}}_{2} \\
* & \breve{\mathcal{P}}-2 \mathscr{W}
\end{array}\right], \quad \hat{\bar{\Sigma}}_{1} \triangleq\left[\begin{array}{ccc}
-\breve{\mathcal{P}} & 0 & \mathscr{L}^{T} \\
* & -\gamma^{2} I & 0 \\
* & * & -I
\end{array}\right], \quad \hat{\bar{\Sigma}}_{2} \triangleq \operatorname{col}\left\{(\mathscr{W} \mathscr{A})^{T},\left(\mathscr{W} \mathscr{B}_{1}\right)^{T}, 0\right\}, \\
& \hat{\overline{\bar{\Sigma}}}_{3} \triangleq \operatorname{col}\left\{\hat{\overline{\mathcal{X}}}^{T}, 0,0,0\right\}, \quad \hat{\bar{\Sigma}}_{4} \triangleq\left[\begin{array}{lllll}
\underline{\mathcal{H}} & \varepsilon_{1}^{(1)} \underline{C}^{T} \quad \underline{\mathcal{H}} & \varepsilon_{2}^{(1)} \underline{D}^{T}
\end{array}\right], \quad \overline{\bar{\Sigma}}_{5} \triangleq\left[\begin{array}{llll}
\overline{\bar{\Sigma}}_{5,1} & \overline{\bar{\Sigma}}_{5,2} & \cdots & \overline{\bar{\Sigma}}_{5, m}
\end{array}\right], \\
& \overline{\bar{\Sigma}}_{5, s} \triangleq\left[\begin{array}{ll}
0 & \epsilon_{s}^{(1)} \underline{C}_{s}^{T}
\end{array}\right], \hat{\overline{\bar{\Sigma}}}_{6} \triangleq\left[\begin{array}{llll}
\hat{\overline{\bar{\Sigma}}}_{6,1} & \hat{\overline{\bar{\Sigma}}}_{6,2} & \cdots & \hat{\overline{\bar{\Sigma}}}_{6, m}
\end{array}\right], \hat{\overline{\bar{\Sigma}}}_{6, s} \triangleq\left[\tilde{\overline{\bar{\beta}}}_{s} \underline{\mathcal{H}}_{s} \quad 0\right], \breve{\mathscr{W}} \triangleq \operatorname{diag}\{\underbrace{\mathscr{W}, \cdots, \mathscr{W}}_{m}\},
\end{aligned}
$$

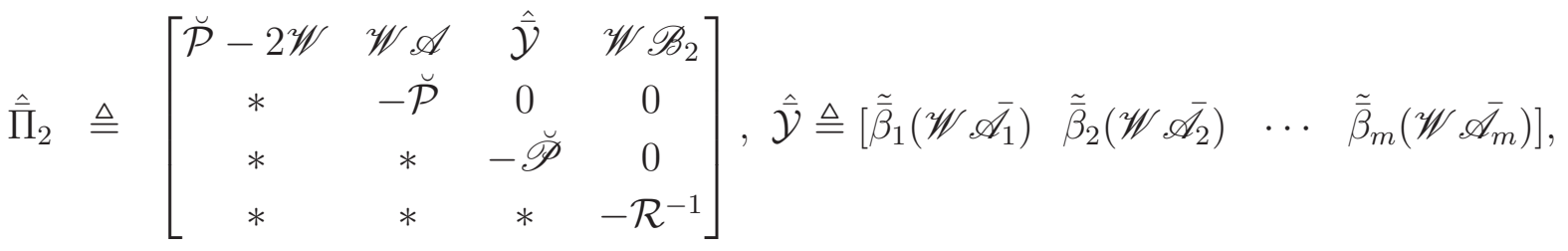

$$
\begin{aligned}
& \bar{M} \triangleq \operatorname{col}\{0, \bar{C}, \underbrace{0, \cdots, 0}_{m+1}\}, \bar{M}_{s} \triangleq \operatorname{col}\{\underbrace{0, \cdots, 0}_{s+1}, \Lambda \bar{C}_{s}, \underbrace{0, \cdots, 0}_{m+1-s}\}, \underline{\hat{U}} \triangleq \operatorname{col}\{-\hat{\mathcal{H}}, \underbrace{0, \cdots, 0}_{m+2}\}, \\
& \hat{\bar{\Pi}}_{3} \triangleq\left[\begin{array}{lll}
\underline{\hat{U}} & \varepsilon_{1}^{(2)} & \bar{M}^{T}
\end{array}\right], \quad \hat{\bar{\Pi}}_{4} \triangleq\left[\begin{array}{llll}
\hat{\bar{\Pi}}_{4,1} & \hat{\bar{\Pi}}_{4,2} & \cdots & \hat{\bar{\Pi}}_{4, m}
\end{array}\right], \quad \hat{\bar{\Pi}}_{4, s} \triangleq\left[\begin{array}{lll}
\tilde{\bar{\beta}}_{s} & \hat{U}^{(2)} & \epsilon_{s}^{(2)} \bar{M}_{s}^{T}
\end{array}\right], \\
& \breve{\varepsilon}^{(1)} \triangleq \operatorname{diag}\left\{\varepsilon_{1}^{(1)}, \varepsilon_{1}^{(1)}, \varepsilon_{2}^{(1)}, \varepsilon_{2}^{(1)}\right\}, \quad \breve{\varepsilon}^{(2)} \triangleq \operatorname{diag}\left\{\varepsilon_{1}^{(2)}, \varepsilon_{1}^{(2)}\right\}, \breve{\epsilon}^{(j)} \triangleq \operatorname{diag}\left\{\bar{\epsilon}_{1}^{(j)}, \bar{\epsilon}_{2}^{(j)}, \cdots, \bar{\epsilon}_{m}^{(j)}\right\}, \\
& \mathcal{R}^{-1} \triangleq \operatorname{diag}\{\underbrace{R^{-1}, \cdots, R^{-1}}_{b}\},(s=1,2, \cdots, m ; j=1,2) \text {. }
\end{aligned}
$$

Theorem 3: For the given disturbance attenuation level $\gamma>0$ and steady-state variance upper bounds $\sigma_{r}^{2}\left(r=1,2, \cdots, n_{x}\right)$, the augmented system (9) is exponentially mean-square stable while achieving the $H_{\infty}$ performance constraint (10) for any nonzero $d\left(t_{k}\right)$ and the steady-state variance constraint (11) for $\bar{\nu}\left(t_{k}\right)$, if there exist matrices $\mathcal{H}_{\varrho}(\varrho=1,2, \cdots, b), W>0$ and $P_{h}>0(h=1,2, \cdots, 2 b)$ such that the following linear matrix inequalities (LMIs) hold:

$$
\begin{aligned}
& {\left[\begin{array}{cccc}
\hat{\bar{\Sigma}}_{1} & \hat{\bar{\Sigma}}_{3} & \hat{\bar{\Sigma}}_{4} & \overline{\bar{\Sigma}}_{5} \\
* & \breve{\mathscr{P}}^{-}-2 \breve{\mathscr{W}} & 0 & \hat{\bar{\Sigma}}_{6} \\
* & * & -\breve{\bar{\varepsilon}}^{(1)} & 0 \\
* & * & * & -\breve{\bar{\epsilon}}^{(1)}
\end{array}\right]<0} \\
& \\
& {\left[\begin{array}{ccc}
\hat{\bar{\Pi}}_{2} & \hat{\bar{\Pi}}_{3} & \hat{\bar{\Pi}}_{4} \\
* & -\breve{\bar{\varepsilon}}^{(2)} & 0 \\
* & * & -\breve{\bar{\epsilon}}^{(2)}
\end{array}\right]<0} \\
& \\
& {\left[\begin{array}{cc}
-\sigma_{r}^{2} & \underline{\mathcal{I}}_{r} \\
* & -P_{\ell}
\end{array}\right]<0\left(r=1,2, \cdots, n_{x} ; \ell=1,2, \cdots, b\right)}
\end{aligned}
$$


Furthermore, if above inequalities are feasible, the desired estimator gains can be determined by

$$
H_{\varrho}=W^{-1} \mathcal{H}_{\varrho}(\varrho=1,2, \cdots, b) .
$$

Proof: By using the Schur Complement Lemma, (12) is equivalent to the following inequality:

$$
\Sigma \triangleq\left[\begin{array}{ccc}
\Sigma_{1} & \Sigma_{2} & \Sigma_{3} \\
* & -\mathcal{P}^{-1} & 0 \\
* & * & -\mathscr{P}^{-1}
\end{array}\right]<0
$$

where

$$
\begin{aligned}
& \Sigma_{1} \triangleq\left[\begin{array}{ccc}
-\mathcal{P} & 0 & \mathscr{L}^{T} \\
* & -\gamma^{2} I & 0 \\
* & * & -I
\end{array}\right], \Sigma_{2} \triangleq \operatorname{col}\left\{\mathscr{A}^{T}+\mathscr{C}^{T}, \mathscr{B}_{1}^{T}+\overline{\mathscr{B}}_{1}^{T}, 0\right\}, \Sigma_{3} \triangleq \operatorname{col}\left\{\mathcal{X}^{T}, 0,0\right\}, \\
& \mathcal{X}^{T} \triangleq\left[\tilde{\bar{\beta}}_{1}\left(\mathscr{A}_{1}^{T}+\overline{\mathscr{C}}_{1}^{T}\right) \quad \tilde{\bar{\beta}}_{2}\left(\mathscr{\mathscr { A }}_{2}^{T}+\overline{\mathscr{C}}_{2}^{T}\right) \ldots \tilde{\bar{\beta}}_{m}\left(\mathscr{\mathscr { A }}_{m}^{T}+\overline{\mathscr{C}}_{m}^{T}\right)\right], \quad \mathscr{P}^{-1} \triangleq \operatorname{diag}\{\underbrace{\mathcal{P}^{-1}, \cdots, \mathcal{P}^{-1}}_{m}\} .
\end{aligned}
$$

In order to cope with the uncertainty factor $F(\bullet)$, we rewrite (30) in the form of (23) as follows:

$$
\begin{aligned}
\bar{\Sigma}+\hat{\bar{H}} F\left(t_{k}\right) \hat{C}+ & \hat{C}^{T} F^{T}\left(t_{k}\right) \hat{\bar{H}}^{T}+\hat{\bar{H}} F\left(t_{k}\right) \hat{D}+\hat{D}^{T} F^{T}\left(t_{k}\right) \hat{\bar{H}}^{T} \\
& +\sum_{s=1}^{m} \tilde{\bar{\beta}}_{s} \hat{\bar{H}}_{s} F\left(t_{k}\right) \hat{C}_{s}+\sum_{s=1}^{m} \tilde{\bar{\beta}}_{s} \hat{C}_{s}^{T} F^{T}\left(t_{k}\right) \hat{\bar{H}}_{s}^{T}<0
\end{aligned}
$$

where

$$
\begin{aligned}
& \bar{\Sigma} \triangleq\left[\begin{array}{ccc}
\Sigma_{1} & \bar{\Sigma}_{2} & \bar{\Sigma}_{3} \\
* & -\mathcal{P}^{-1} & 0 \\
* & * & -\mathscr{P}^{-1}
\end{array}\right], \quad \bar{\Sigma}_{2} \triangleq \operatorname{col}\left\{\mathscr{A}^{T}, \mathscr{B}_{1}^{T}, 0\right\}, \bar{\Sigma}_{3} \triangleq \operatorname{col}\left\{\overline{\mathcal{X}}^{T}, 0,0\right\},
\end{aligned}
$$

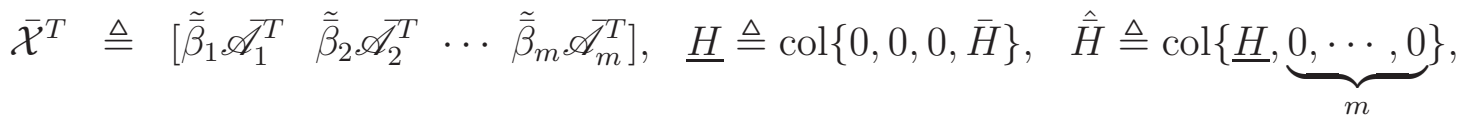

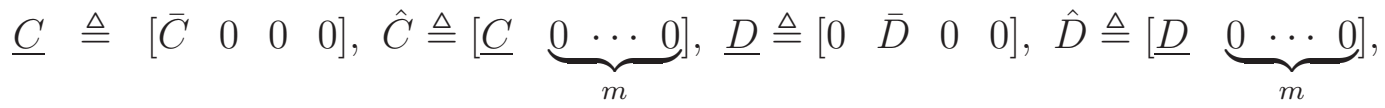

$$
\begin{aligned}
& \underline{H}_{s} \triangleq \operatorname{col}\{\underbrace{0, \cdots, 0}_{s-1}, \bar{H}, \underbrace{0, \cdots, 0}_{m-s}\}, \quad \hat{\bar{H}}_{s} \triangleq \operatorname{col}\left\{0,0,0,0, \underline{H}_{s}\right\}, \\
& \underline{C}_{s} \triangleq\left[\begin{array}{llll}
\Lambda \bar{C}_{s} & 0 & 0 & 0
\end{array}\right], \hat{C}_{s} \triangleq[\underline{C}_{s} \underbrace{0 \cdots 0}_{m}],(s=1,2, \cdots, m) .
\end{aligned}
$$

Applying Lemma 1 to (31), it follows that (31) holds if and only if there exist positive scalars $\varepsilon_{1}^{(1)}, \varepsilon_{2}^{(1)}, \epsilon_{s}^{(1)}(s=1,2, \cdots, m)$ such that the following matrix inequality holds

$$
\overline{\bar{\Sigma}} \triangleq\left[\begin{array}{cccc}
\overline{\bar{\Sigma}}_{1} & \overline{\bar{\Sigma}}_{3} & \overline{\bar{\Sigma}}_{4} & \overline{\bar{\Sigma}}_{5} \\
* & -\mathscr{P}^{-1} & 0 & \overline{\bar{\Sigma}}_{6} \\
* & * & -\breve{\bar{\varepsilon}}^{(1)} & 0 \\
* & * & * & -\overline{\bar{\epsilon}}^{(1)}
\end{array}\right]<0
$$

where

$$
\overline{\bar{\Sigma}}_{1} \triangleq\left[\begin{array}{cc}
\bar{\Sigma}_{1} & \bar{\Sigma}_{2} \\
* & -\mathcal{P}^{-1}
\end{array}\right], \quad \bar{\Sigma}_{3} \triangleq \operatorname{col}\left\{\bar{\Sigma}_{3}, 0\right\}, \quad \overline{\bar{\Sigma}}_{4} \triangleq\left[\begin{array}{llll}
\underline{H} & \varepsilon_{1}^{(1)} \underline{C}^{T} & \underline{H} & \varepsilon_{2}^{(1)} \underline{D^{T}}
\end{array}\right]
$$




$$
\overline{\bar{\Sigma}}_{6} \triangleq\left[\begin{array}{llll}
\overline{\bar{\Sigma}}_{6,1} \quad \overline{\bar{\Sigma}}_{6,2} \cdots \overline{\bar{\Sigma}}_{6, m}
\end{array}\right], \quad \overline{\bar{\Sigma}}_{6, s} \triangleq\left[\begin{array}{ll}
\tilde{\bar{\beta}}_{s} \underline{H}_{s} & 0
\end{array}\right], \quad(s=1,2, \cdots, m) .
$$

After using Schur Complement Lemma to (16), we have

$$
\Pi_{1} \triangleq\left[\begin{array}{cccc}
-\mathcal{Q} & \mathscr{A}+\mathscr{C} & \mathcal{Y} & \mathscr{B}_{2} \\
* & -\mathcal{Q}^{-1} & 0 & 0 \\
* & * & -\mathscr{Q}^{-1} & 0 \\
* & * & * & -\mathcal{R}^{-1}
\end{array}\right]<0
$$

where $\mathscr{Q}^{-1} \triangleq \operatorname{diag}\{\underbrace{\mathcal{Q}^{-1}, \cdots, \mathcal{Q}^{-1}}_{\ell+1}\}, \mathcal{Y} \triangleq\left[\tilde{\bar{\beta}}_{1}\left(\overline{\mathscr{A}}_{1}+\overline{\mathscr{C}}_{1}\right) \quad \tilde{\bar{\beta}}_{2}\left(\overline{\mathscr{A}}_{2}+\overline{\mathscr{C}}_{2}\right) \quad \cdots \quad \tilde{\bar{\beta}}_{m}\left(\overline{\mathscr{A}}_{m}+\overline{\mathscr{C}}_{m}\right)\right]$.

Similarly, by denoting $\mathcal{Q}=\mathcal{P}^{-1}$, we rewrite (33) in the form of (23) as follows:

$$
\Pi_{2}+\underline{U} F\left(t_{k}\right) \bar{M}+\bar{M}^{T} F^{T}\left(t_{k}\right) \underline{U}^{T}+\sum_{s=1}^{m} \tilde{\bar{\beta}}_{s} \underline{U} F\left(t_{k}\right) \bar{M}_{s}+\sum_{s=1}^{m} \tilde{\bar{\beta}}_{s} \bar{M}_{s}^{T} F^{T}\left(t_{k}\right) \underline{U}^{T}<0
$$

where

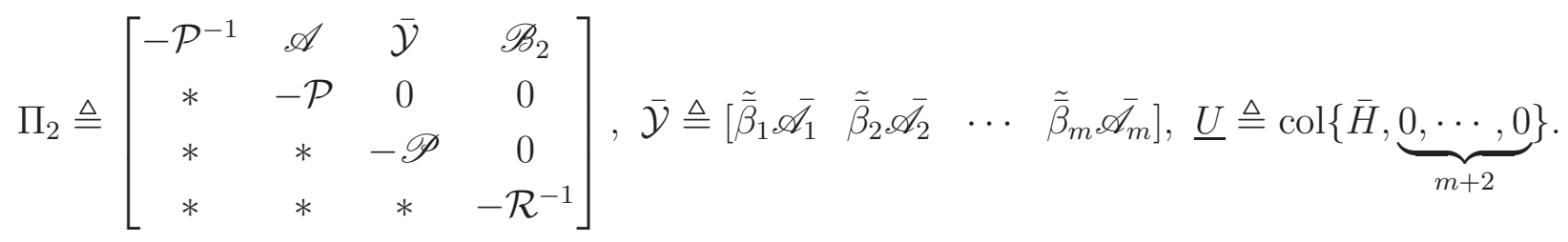

By applying Lemma 1 again to (34), we know that (34) holds if and only if there exist positive scalars $\varepsilon_{1}^{(2)}, \epsilon_{s}^{(2)}(s=1,2, \cdots, m)$ such that the following LMI holds:

$$
\bar{\Pi}_{2} \triangleq\left[\begin{array}{ccc}
\Pi_{2} & \Pi_{3} & \Pi_{4} \\
* & -\breve{\bar{\varepsilon}}^{(2)} & 0 \\
* & * & -\breve{\bar{\epsilon}}^{(2)}
\end{array}\right]<0
$$

where

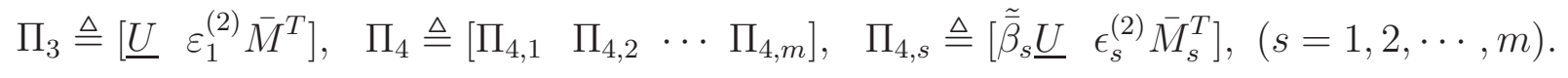

To this end, in order to design the estimator by Matlab LMI Toolbox to effectively, we assume $\breve{\mathcal{P}} \triangleq$ $\operatorname{diag}\left\{P_{1}, P_{2}, \cdots, P_{2 b}\right\}$ and let $\mathcal{H}_{\varrho} \triangleq W H_{\varrho}(\varrho=1,2, \cdots, b)$. By noting $W>0$ and $P_{h}>0(h=$ $1,2, \cdots, 2 b)$, we have $\left(P_{h}-W\right) P_{h}^{-1}\left(P_{h}-W\right) \geq 0$, which is equivalent to

$$
-W P_{h}^{-1} W \leq P_{h}-2 W \quad(h=1,2, \cdots, 2 b)
$$

Applying the congruence transformation $\operatorname{diag}\{I, I, I, \underbrace{\mathscr{W}, \cdots, \mathscr{W}}_{m+1}, \underbrace{I, \cdots, I}_{2 m+4}\}$ to (32), we get (26). Further applying the congruence transformations $\operatorname{diag}\{\mathscr{W}, \underbrace{I, \cdots, I}_{3 m+b+2}\}$ to (35), we obtain (27). At the same time, the estimator gain can be expressed as (29).

On the other hand, from $\mathcal{Q}=\mathcal{P}^{-1}$, we assume $\breve{\mathcal{Q}} \triangleq \breve{\mathcal{P}}^{-1} \triangleq \operatorname{diag}\left\{P_{1}^{-1}, P_{2}^{-1}, \cdots, P_{2 b}^{-1}\right\}$, and rewrite (17) as follows:

$$
\underline{\mathcal{I}}_{r} \overline{\mathcal{I}}_{\ell} \breve{\mathcal{Q}}_{\ell} \overline{\mathcal{I}}_{\ell}^{T} \underline{\mathcal{I}}_{r}^{T}=\underline{\mathcal{I}}_{r} \overline{\mathcal{I}}_{\ell} \breve{\mathcal{P}}^{-1} \overline{\mathcal{I}}_{\ell}^{T} \underline{\mathcal{I}}_{r}^{T}=\underline{\mathcal{I}}_{r} P_{\ell}^{-1} \underline{\mathcal{I}}_{r}^{T} \leq \sigma_{r}^{2}\left(r=1,2, \cdots, n_{x} ; \quad \ell=1,2, \cdots, b\right)
$$

by using Schur Complement Lemma to (36), we have (28), which concludes the proof from Theorems 1 and 2. 
Remark 3: In this paper, the variance-constrained state estimation problem is investigated for a class of NMSs with quantization and probabilistic sensor failures. The main features of our results are twofold: i) the quantified relationships have been established among the $H_{\infty}$ performance level, the upper bounds on the steady-state variances of the estimation errors, the quantizer parameters, the sensor failure probabilities and the multi-rate multiple $b$ of the sampling period $h$; and ii) the proposed approach has offered much flexibility in making compromise between the steady-state variances and the $H_{\infty}$ performance, while the essential multiple objectives can all be achieved simultaneously in the framework of NMSs.

In order to show the combined effect of the considered variance constraints, quantizer parameters, sensor failure probabilities and the multi-rate sampling, we now discuss the following two optimization problems for given quantization density $\chi^{(s)}(s=1,2, \cdots, m)$, multi-rate multiple $b$, sensor failure parameters $\bar{\beta}_{s}$ and $\tilde{\bar{\beta}}_{s}^{2}(s=1,2, \cdots, m)$.

P1: For given steady-state estimation error variance-constrained bounds $\sigma_{1}^{2}, \cdots, \sigma_{n_{x}}^{2}$, the optimal $H_{\infty}$ estimator design problem:

$$
\min _{W, \mathcal{H}_{1}, \cdots, \mathcal{H}_{b}, P_{1}, \cdots, P_{2 b}} \gamma^{2} \text { subject to }(26)-(28) .
$$

P2: For given $H_{\infty}$ performance level $\gamma$, the minimum weighted variance-constrained estimator design problem:

$$
\min _{W, \mathcal{H}_{1}, \cdots, \mathcal{H}_{b}, P_{1}, \cdots, P_{2 b}} \sum_{r=1}^{n_{x}} c_{r} \sigma_{r}^{2} \text { subject to }(26)-(28) .
$$

where $c_{r}\left(r=1,2, \cdots, n_{x}\right)$ are given weighting coefficients for variances and satisfy $\sum_{r=1}^{n_{x}} c_{r}=1$.

\section{A NUMERICAL EXAMPLE}

In this section, similar to [20], [37], a maneuvering target tracking system is presented to demonstrate the effectiveness of the proposed design scheme, and the involved system has the following state-space model:

$$
\begin{aligned}
x\left(T_{k+1}\right) & =\left[\begin{array}{cc}
0.3 & h \\
0 & 0.4
\end{array}\right] x\left(T_{k}\right)+\left[\begin{array}{c}
\frac{h}{2} \\
0.1
\end{array}\right] \omega\left(T_{k}\right)+\left[\begin{array}{c}
0.1 \\
\frac{h^{2}}{2}
\end{array}\right] \nu\left(T_{k}\right) \\
z\left(T_{k}\right) & =\left[\begin{array}{ll}
0.5 & 0.4
\end{array}\right] x\left(T_{k}\right)
\end{aligned}
$$

where $h$ is the sampling period. $x\left(T_{k}\right)=\operatorname{col}\left\{x_{p}\left(T_{k}\right), x_{v}\left(T_{k}\right)\right\}$ is the system state, $x_{p}\left(T_{k}\right)$ and $x_{v}\left(T_{k}\right)$ are the position and velocity of the target at time $T_{k}$, respectively.

In a networked maneuvering target tracking system where the sensor signals are transmitted through communication networks, it is often the case that the measurement outputs are quantized before being transmitted to the estimator. At the same time, the measurements received by sensors could be neither completely missing nor completely successful, but only part of the information can go through. Suppose that only the position of the maneuvering target is measurable. Then, we use the following equation to model the measurements with quantization effects and probabilistic sensor failures at time $t_{k}$ :

$$
\bar{y}\left(t_{k}\right)=q\left(\beta\left(t_{k}\right)\left[\begin{array}{ll}
1 & 0
\end{array}\right] x\left(t_{k}\right)+0.5 \xi\left(t_{k}\right)\right)
$$


The parameters of the logarithmic quantizer $q(\cdot)$ are chosen as $u_{0}=2, \chi=0.4$, and the probability density function of sensor failure coefficient is taken as

$$
f\left(\beta\left(t_{k}\right)\right)=\left\{\begin{array}{cc}
10 \beta\left(t_{k}\right), & 0 \leq \beta\left(t_{k}\right) \leq 0.20 \\
-2.50\left(\beta\left(t_{k}\right)-1\right), & 0.20<\beta\left(t_{k}\right) \leq 1 .
\end{array}\right.
$$

then the mathematical expectation $\bar{\beta}$ and the variance $\tilde{\bar{\beta}}^{2}$ can be calculated as 0.4000 and 0.0467 , respectively.

Here, the sampling period $h$ is set as $0.5 s$ and the variance of Gaussian white noise $\nu\left(T_{k}\right)$ is taken as $R=0.3$. The disturbance input $\omega\left(T_{k}\right)$ and the measurement noise $\xi\left(t_{k}\right)$ are chosen as following:

$$
\omega\left(T_{k}\right)=0.1 \mathrm{e}^{-0.05 T_{k}} \sin \left(T_{k}\right), \xi\left(t_{k}\right)=\frac{\mathrm{e}^{-0.05 t_{k}}}{0.2 t_{k}+1} .
$$

Actually, the system under consideration is a two-rate sampled-data one, that is, the state estimation for both the position and the velocity is conducted at a fast rate with the period $h$, while the sensor samples the target position at a slow one with the period $b h$. We aim to design the estimator, by using the quantized measurement, to estimate the state (position) of the maneuvering target subject to bounded energy disturbance and Gaussian white noise. Now, let us examine the following two cases.

Case 1. The variance constraints on the steady-state estimation error are set as $\sigma_{1}=0.6$ and $\sigma_{2}=0.4$. By using the MATLAB LMI toolbox and considering the optimization problem (P1), we obtain the minimum disturbance attenuation level $\gamma$ and corresponding estimator gains $H_{\varrho}(\varrho=1,2, \cdots, b)$ in Table I with different multi-rate multiple $b$. Take the initial state of (1) and its estimation as $x\left(T_{0}\right)=\operatorname{col}\{-0.1,0.1\}$ and $\hat{x}\left(t_{0}\right)=\operatorname{col}\{-0.2,0.2\}$, respectively. The estimated error $e_{z}\left(t_{k}\right)$ for the position of the maneuvering target is plotted in Fig.2.

TABLE I

\begin{tabular}{|c|c|c|c|c|c|c|c|c|c|c|c|}
\hline \multirow[b]{2}{*}{$b=2$} & \multirow{2}{*}{$\begin{array}{c}\gamma \\
0.7734\end{array}$} & \multicolumn{10}{|c|}{$H_{\varrho}$} \\
\hline & & & & & $H_{1}=$ & $\begin{array}{c}0.0288 \\
-0.0770 \\
\end{array}$ &,$H_{2}=$ & $\left.\begin{array}{c}-0.0040 \\
0.0822\end{array}\right]$ & & & \\
\hline$b=3$ & 0.8073 & & & $H_{1}=$ & $\begin{array}{c}0.0105 \\
-0.0291 \\
\end{array}$ &,$H_{2}=$ & $\begin{array}{c}0.0009 \\
-0.0026 \\
\end{array}$ &,$H_{3}=$ & $\begin{array}{l}0.0724 \\
0.0715 \\
\end{array}$ & & \\
\hline$b=4$ & 0.8472 & & $H_{1}=$ & \begin{tabular}{c|}
0.0066 \\
-0.0186 \\
\end{tabular} &,$H_{2}=$ & $\begin{array}{c}0.0007 \\
-0.0019 \\
\end{array}$ &,$H_{3}=$ & $\begin{array}{c}0.0428 \\
-0.0055 \\
\end{array}$ &,$H_{4}=$ & $\begin{array}{l}0.0229 \\
0.0346\end{array}$ & \\
\hline$b=5$ & 0.8728 & $H_{1}=$ & $\begin{array}{c}0.0096 \\
-0.0271\end{array}$ &,$H_{2}=$ & $\begin{array}{c}0.0004 \\
-0.0011\end{array}$ &,$H_{3}=$ & $\begin{array}{c}0.0266 \\
-0.0159\end{array}$ & $9, H_{4}=$ & $\begin{array}{l}0.0190 \\
0.0070\end{array}$ &,$H_{5}=$ & $\begin{array}{l}0.0146 \\
0.0121\end{array}$ \\
\hline
\end{tabular}

THE PERMITTED MINIMUM $\gamma$ AND CORRESPONDING ESTIMATOR GAINS $H_{\varrho}(\varrho=1,2, \cdots, b)$.

Case 2. For the given $H_{\infty}$ performance level $\gamma=0.95$ and weighting coefficients $c_{1}=0.4, c_{1}=0.6$, we now deal with the problem (P2). Solving the optimization problem (38), we obtain the minimum individual variance values $\sigma_{r}(r=1,2)$ and corresponding estimator gains $H_{\varrho}(\varrho=1,2, \cdots, b)$ in Table II. Choosing the same initial values as Case I, the simulation results are shown in Figs. 3-4, which display the actual steady-state variance for $e_{1}\left(t_{k}\right)=x_{1}\left(t_{k}\right)-\hat{x}_{1}\left(t_{k}\right)$ and $e_{2}\left(t_{k}\right)=x_{2}\left(t_{k}\right)-\hat{x}_{2}\left(t_{k}\right)$, respectively.

Tables I-II demonstrate the relationship between $H_{\infty}$ performance level $\gamma$ and variance upper bounds $\sigma_{r}(r=1,2)$ as well as the multi-rate multiple $b$ of the sampling period $h$. It can be observed from Table 


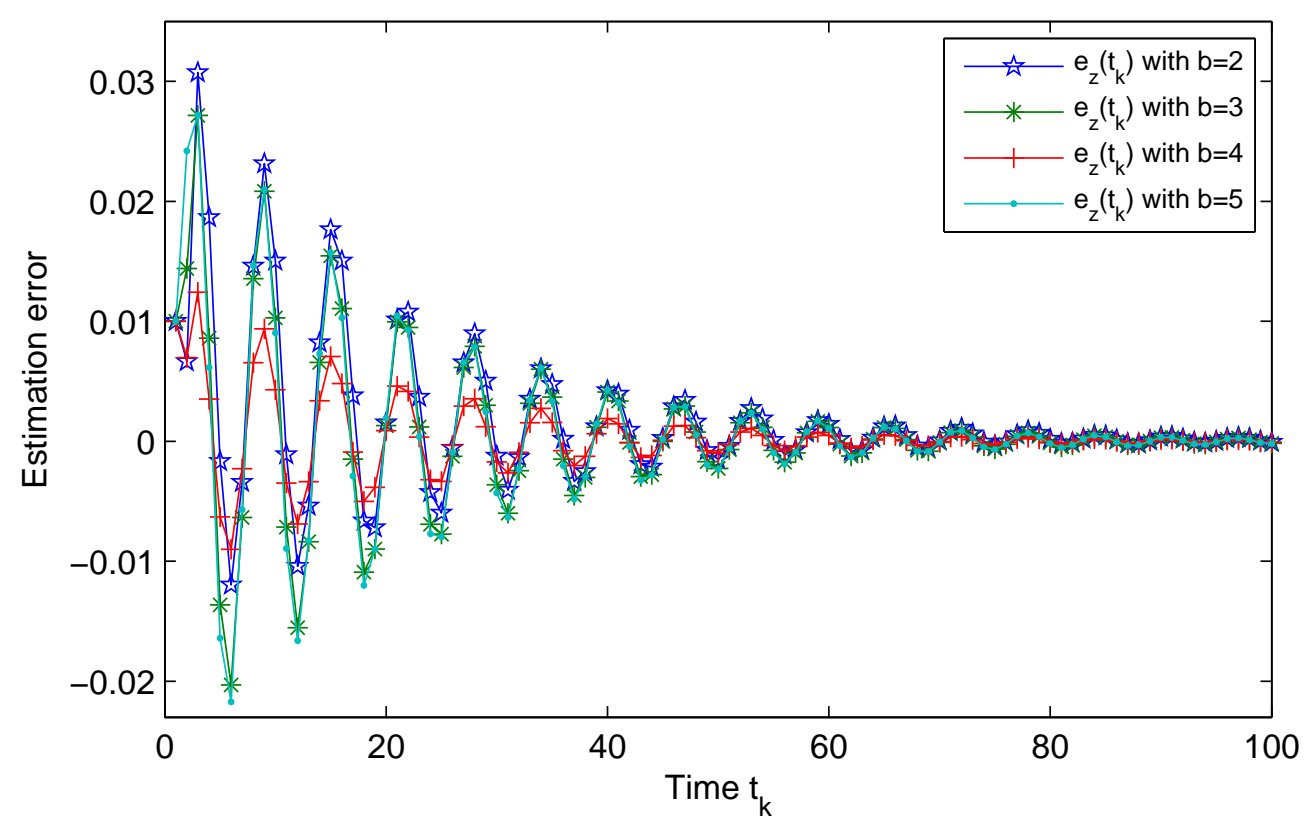

Fig. 2. Estimation error $e_{z}\left(t_{k}\right)$ for different $b$.

TABLE II

THE MiNIMUM VARIANCE VALUES $\sigma_{r}(r=1,2)$ AND CORRESPONDING ESTIMATOR GAINS $H_{\varrho}(\varrho=1,2, \cdots, b)$.

\begin{tabular}{l|ccc|c|c|c|c}
\hline & $\sigma_{1}$ & $\sigma_{2}$ & $H_{\varrho}$ \\
\hline$b=2$ & 0.2368 & 0.0341 & $\left.\left.H_{1}=\begin{array}{c}0.0814 \\
-0.2221\end{array}\right], H_{2}=\begin{array}{c}-0.0221 \\
0.1561\end{array}\right]$ \\
\hline$b=3$ & 0.2759 & 0.0389 & $\left.\left.H_{1}=\begin{array}{c}0.0323 \\
-0.0907\end{array}\right], H_{2}=\begin{array}{c}0.0018 \\
-0.0051\end{array}\right], H_{3}=\left[\begin{array}{l}0.0497 \\
0.0913\end{array}\right]$ \\
\hline$b=4$ & 0.3011 & 0.0418 & $H_{1}=\left[\begin{array}{c}0.0252 \\
-0.0712\end{array}\right], H_{2}=\left[\begin{array}{c}0.0008 \\
-0.0022\end{array}\right], H_{3}=\left[\begin{array}{l}0.0254 \\
0.0245\end{array}\right], H_{4}=\left[\begin{array}{l}0.0194 \\
0.0336\end{array}\right]$ \\
\hline$b=5$ & 0.3166 & 0.0436 & $H_{1}=\left[\begin{array}{c}0.0223 \\
-0.0631\end{array}\right], H_{2}=10^{-3} \times\left[\begin{array}{c}0.2863 \\
-0.8104\end{array}\right], H_{3}=\left[\begin{array}{c}0.0154 \\
0.0083\end{array}\right], H_{4}=\left[\begin{array}{c}0.0135 \\
0.0142\end{array}\right], H_{5}=\left[\begin{array}{l}0.0108 \\
0.0168\end{array}\right]$ \\
\hline
\end{tabular}

I and Table II that, with increased $b$, the disturbance attenuation performance deteriorates and the variance upper bounds become bigger, and these observations can also been confirmed from Figs. 3-4, which are in agreement with the engineering practice.

\section{CONCLUSION}

In this paper, the variance-constrained $H_{\infty}$ state estimation problem has been investigated for a class of networked multi-rate systems. The system under consideration involves network-induced probabilistic sensor failures and measurement quantization. The state estimator has been designed such that both $H_{\infty}$ performance and variance constraints on steady-state estimation error are achieved. By utilizing the stochastic analysis techniques, sufficient conditions have been established in the form of matrix inequalities reflecting the relationship among $H_{\infty}$ performance level, variance upper bounds, quantizer parameters, sensor failure parameters and multi-rate multiple. The estimator gains matrix have been characterized by means of the feasibility of certain matrix inequalities. Two additional optimization problems have 


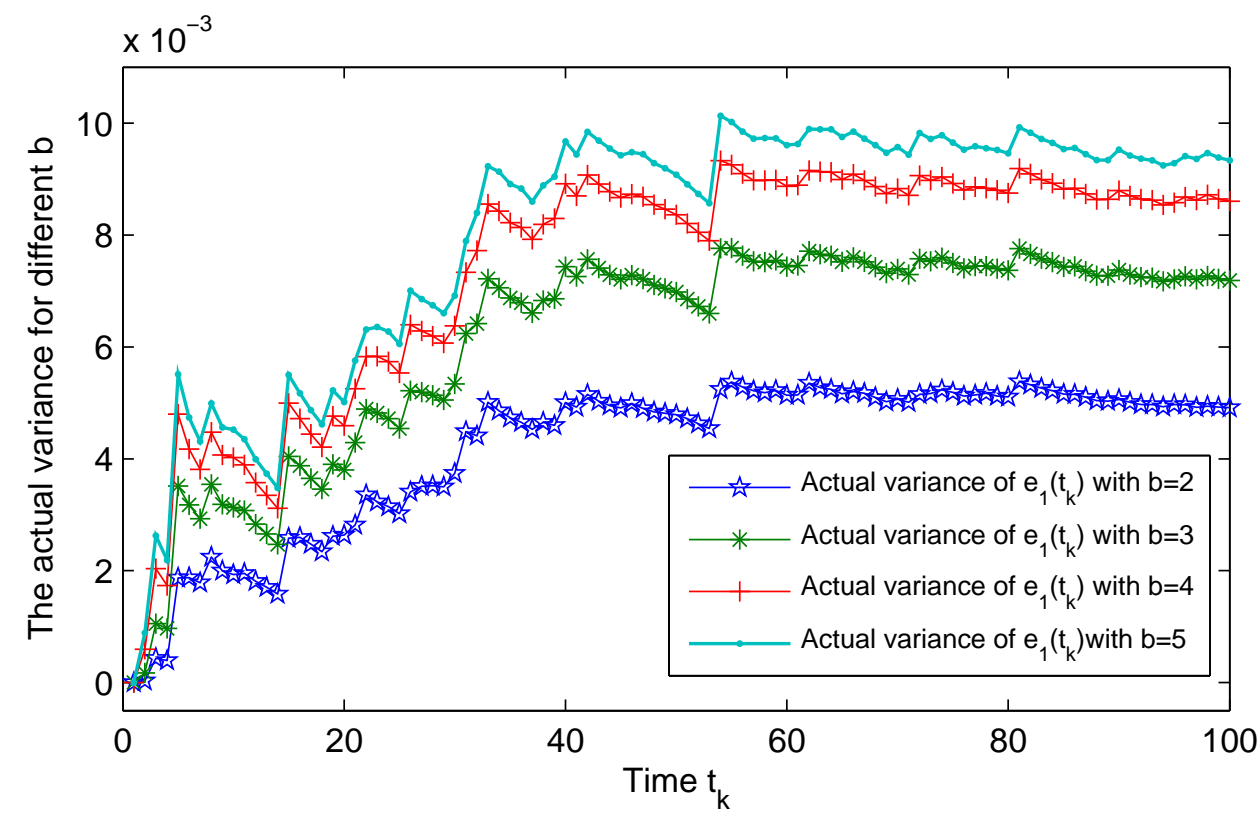

Fig. 3. The actual steady-state estimation error variance for $e_{1}\left(t_{k}\right)$ for different $b$.

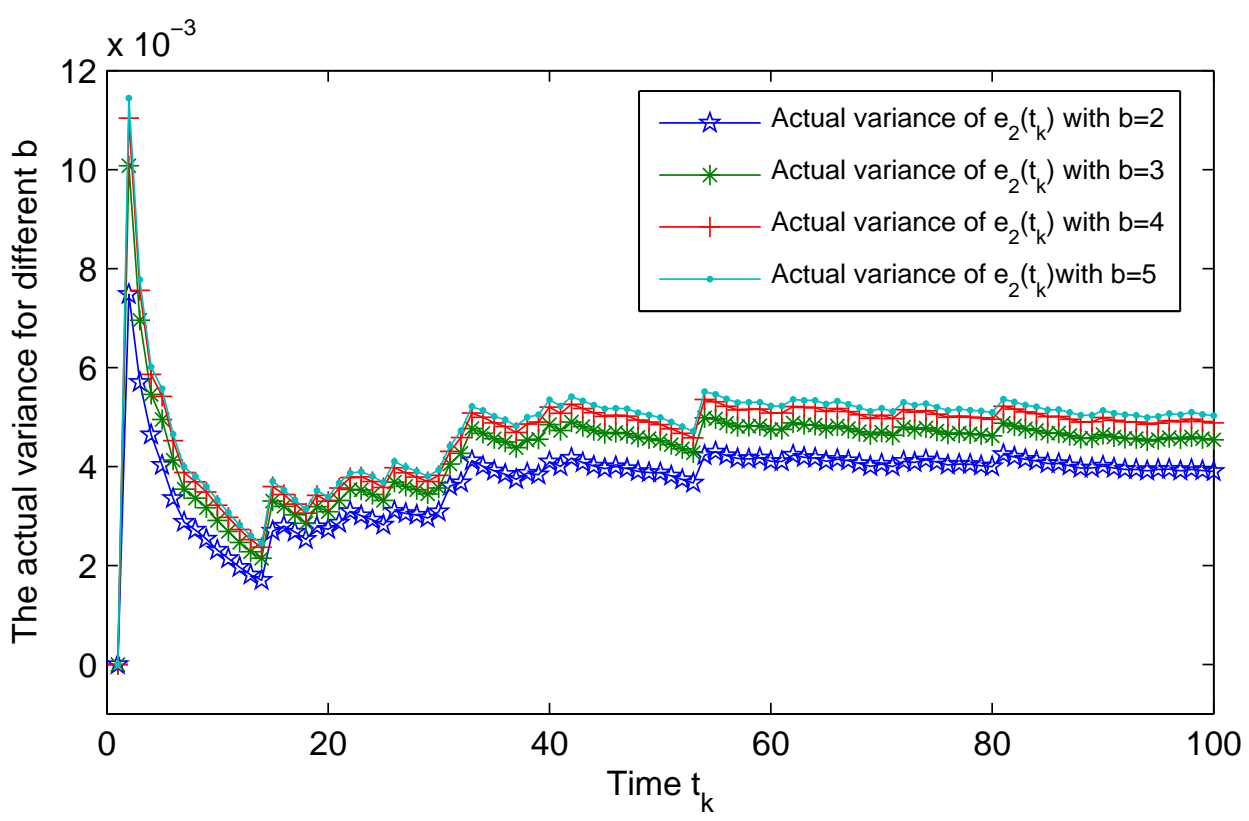

Fig. 4. The actual steady-state estimation error variance for $e_{2}\left(t_{k}\right)$ for different $b$.

been considered with respect to the $H_{\infty}$ performance index and the weighted error variances. Finally, a simulation example has been provided to show the effectiveness of the proposed estimator design scheme.

\section{REFERENCES}

[1] M. Basin, S. Elvira-Ceja and E. Sanchez. Mean-square $H_{\infty}$ filtering for stochastic systems: application to a 2DOF helicopter. Signal Processing, Vol. 92, No. 3, pp. 801-806, 2012.

[2] S. Boyd, L. E. Ghaoui, E. Feron, and V. Balakrishnan, Linear matrix inequalities in system and control theory. Philadelphia, PA: SIAM Stud. Appl. Math., 1994.

[3] R. Caballero-Águila, A. Hermoso-Carazo and J. Linares-Pérez. Optimal state estimation for networked systems with random parameter matrices, correlated noises and delayed measurements. International Journal of General Systems Vol. 44, No. 2, pp. 142-154, 2015. 
[4] M. Cloosterman, N. Wouw, W. Heemels and H. Nijmeijer, Stability of networked control systems with uncertain time-varying delays, IEEE Transactions on Automatic Control, Vol. 54, No. 7, pp. 1575-1580, 2009.

[5] H. Dong, Z. Wang, S. X. Ding and H. Gao, Finite-horizon reliable control with randomly occurring uncertainties and nonlinearities subject to output quantization, Automatica, Vol. 52, pp. 355-362, 2015.

[6] H. Dong, Z. Wang, D.W.C. Ho and H. Gao, Variance-constrained $H_{\infty}$ filtering for nonlinear time-varying stochastic systems with multiple missing measurements: the finite-horizon case, IEEE Transactions on Signal Processing, Vol. 58, No. 5, pp. 2534-2543, 2010.

[7] H. Dong, Z. Wang, F. E. Alsaadi and B. Ahmad, Event-triggered robust distributed state estimation for sensor networks with statedependent noises, International Journal of General Systems, Vol. 44, No. 2, pp. 254-266, 2015.

[8] D. Ding, Z. Wang, J. Lam and B. Shen, Finite-Horizon $H_{\infty}$ control for discrete time-varying systems with randomly occurring nonlinearities and fading measurements, IEEE Transactions on Automatic Control, Vol. 60, No. 9, pp. 2488-2493, 2015.

[9] D. Ding, Z. Wang, B. Shen and H. Dong, Envelope-constrained H-infinity filtering with fading measurements and randomly occurring nonlinearities: the finite horizon case, Automatica, Vol. 55, pp. 37-45, 2015.

[10] D. Ding, Z. Wang and B. Shen, Recent advances on distributed filtering for stochastic systems over sensor networks, International Journal of General Systems, Vol. 43, No. 3-4, pp. 372-386, 2014.

[11] D. Ding, Z. Wang, Fuad E. Alsaadi, and Bo Shen, Receding horizon filtering for a class of discrete time-varying nonlinear systems with multiple missing measurements, International Journal of General Systems, Vol. 44, No. 2, pp. 198-211, 2015.

[12] M. Fu and L. Xie. The sector bound approach to quantized feedback control, IEEE Transactions on Automatic Control, Vol. 50, No. 11, pp. 1698-1711, 2005.

[13] J. Hu, J. Liang, D. Chen, D. Ji and J. Du, A recursive approach to non-fragile filtering for networked systems with stochastic uncertainties and incomplete measurements, The Journal of The Franklin Institute, Vol. 352, No. 5, pp. 1946-1962, 2015.

[14] Y. Hung and F. Yang, Robust $H_{\infty}$ filtering with error variance constraints for uncertain discrete time-varying systems with uncertainty, Automatica, Vol. 39, No. 7, pp. 1185-1194, 2003.

[15] G. Heredia, A. Ollero, M. Bejar and R. Mahtani, Sensor and actuator fault detection in small autonomous helicopters, Mechatronics, Vol.18, No.2, pp. 90-99, 2008.

[16] H. Ishii and T. Basar, Remote control of LTI systems over networks with state quantization, Systems and Control Letters, Vol. 54, No. 1, pp. 15-31, 2005.

[17] I. Izadi, Q. Zhao and T. Chen, An optimal scheme for fast rate fault detection based on multi-rate sampled data, Journal of Process Control, Vol. 15, No. 3, pp. 307-319, 2005.

[18] J. Kullaa, Detection, identification, and quantification of sensor fault in a sensor network, Mechanical Systems and Signal Processing, Vol.40, No.1, pp. 208-221, 2013.

[19] D. Liberzon, Hybrid feedback stabilization of systems with quantized signals, Automatica, Vol. 39, No. 9, pp. 1543-1554, 2003.

[20] Y. Liang, T. Chen and Q. Pan, Multi-rate stochastic $H_{\infty}$ filtering for networked multi-sensor fusion, Automatica, Vol. 46, No. 2, pp. 437-444, 2010.

[21] K. Liu, E. Fridman and K. H. Johanssonc, Dynamic quantization of uncertain linear networked control systems, Automatica, Vol. 59, pp. 248-255, 2015.

[22] G. Liu, J. Mu, D. Rees and S. Chai, Design and stability analysis of networked control systems with random communication time delay using the modified MPC, International Journal of Control, Vol. 79, No. 4, pp. 288-297, 2006.

[23] Y. Liu, F. E. Alsaadi, X. Yin and Y. Wang, Robust $H_{\infty}$ filtering for discrete nonlinear delayed stochastic systems with missing measurements and randomly occurring nonlinearities, International Journal of General Systems, Vol. 44, No. 2, pp. 169-181, 2015.

[24] Y. Luo, G. Wei, Y. Liu and X. Ding, Reliable $H_{\infty}$ state estimation for 2-D discrete systems with infinite distributed delays and incomplete observations, International Journal of General Systems, Vol. 44, No. 2, pp. 155-168, 2015.

[25] H. Matsuura and K. Wasaki, Quantum theory of fundamental network (path integral expression circuits and network's quantization), International Journal of Innovative Computing Information and Control, Vol.10, No.5, pp.1601-1623, 2014.

[26] L. Ma, Z. Wang and Y. Bo, Variance-Constrained Multi-Objective Stochastic Control and Filtering, John Wiley \& Sons, Chichester, 336 pages, 2015. (ISBN: 978-1-118-92949-0)

[27] L. Ma, Z. Wang, J. Hu, Y. Bo and Z. Guo, Robust variance-constrained filtering for a class of nonlinear stochastic systems with missing measurements, Signal Processing, Vol. 90, No. 6, pp. 2060-2071, 2010.

[28] Y. Niu and D.W.C. Ho, Control strategy with adaptive quantizers parameters under digital communication channels, Automatica, Vol. 50, No. 10, pp. 2665-2671, 2014.

[29] C. Peng, Q. Han and D. Yue, Communication-delay-distribution-dependent decentralized control for large-scale systems with IP-based communication networks, IEEE Transactions on Control Systems Technology, Vol. 21, No. 3, pp. 820-830, 2013.

[30] L. Qiu and T. Chen, $H_{2}$-optimal design of multirate sampled-data systems, IEEE Transactions on Automatic Control, Vol. 39, No. 12, pp. 2506-2511, 1994.

[31] P. Shi, M. Liu and L. Zhang, Fault-tolerant sliding mode observer synthesis of Markovian jump systems using quantized measurements, IEEE Trans on Industrial Electronics, Vol.62, No.9, pp. 5910-5918, 2015. 
[32] A. Subramanian and A.H. Sayed, Multiobjective filter design for uncertain stochastic time-delay systems, IEEE Transactions on Automatic Control, Vol. 49, No. 1, pp. 149-154, 2004.

[33] L. Sheng, W. Zhang and M. Gao, Relationship between Nash equilibrium strategies and $H_{\infty} / H_{2}$ control of stochastic Markov jump systems with multiplicative noise, IEEE Transactions on Automatic Control, Vol. 59, No. 9, pp. 2592-2597, 2014.

[34] Y. Wang, S. Ding, H. Ye and G. Wang, A new fault detection scheme for networked control systems subject to uncertain time-varying delay, IEEE Transactions on Signal Processing, Vol. 56, No. 10, pp. 5258-5268, 2008.

[35] Z. Wang, D.W.C. Ho and X. Liu, Variance-constrained filtering for uncertain stochastic systems with missing measurements, IEEE Transactions on Automatic Control, Vol. 48, No. 7, pp. 560-567, 2003.

[36] Z. Wang, F. Yang, D. W. C. Ho and X. Liu, Robust $H_{\infty}$ control for networked systems with random packet losses, IEEE Transactions on Systems, Man, and Cybernetics - Part B, Vol. 37, No. 4, pp. 916-924, 2007.

[37] W. Zhang, G. Feng and L. Yu, Multi-rate distributed fusion estimation for sensor networks with packet losses, Automatica, Vol. 48, No. 9, pp. 2016-2028, 2012.

[38] Y. Zhang, Z. Liu, H. Fang and H. Chen, $H_{\infty}$ fault detection for nonlinear networked systems with multiple channels data transmission pattern, Information Sciences, Vol. 221, No. 1, pp. 534-543, 2013. 